\title{
Direct and Highly Efficient Estimation of the Entropy through the Canonical Ensemble Average of Acceptance Ratios
}

\author{
Seung Do Hong, ${ }^{\dagger}$ Hang-Cheol Shin, and Kwang-Hwi Cho* \\ ${ }^{\dagger}$ CAMD Research Center, Soongsil University, Seoul 156-743, Korea \\ Department of Bioinformatics and Life Science and CAMD Research Center, Soongsil University, Seoul 156-743, Korea \\ *E-mail: chokh@ssu.ac.kr \\ Received July 18, 2005
}

Key Words : Acceptance ratio, Direct calculation, Entropy

The evaluation of entropy is an important topic in relation to the direction of spontaneous change. A variety of methods for the calculation of the entropy and free energy have been proposed. ${ }^{1-3}$ However, the calculation of the entropy through computer simulation has posed difficulties because the entropy is not a mechanical property but a statistical property. The difficulties are related to large fluctuations attendant upon the averages of the Boltzmann factors.

The particle insertion (PI) method provides a simple way of calculating chemical potentials. ${ }^{4}$ In this method, the excess chemical potential $\mu^{e x}$ is expressed as

$$
\frac{\mu^{e x}}{k T}=-\ln \left\langle\exp \left(\frac{-\Delta \Phi}{k T}\right)\right\rangle
$$

where $k$ is the Boltzmann constant, $T$ is the absolute temperature, and $\Delta \Phi$ is the potential energy change which would result from the insertion of a particle into an $N$ particle system. Here, $\langle\cdots\rangle$ denotes the canonical ensemble average. In this method, the test particle is added at random. This method is hard to apply at high densities due to extremely low acceptance ratios of the inserted particles. The cavity insertion method modified from the PI method has been applied to the calculation of the free energy profile of several small molecules in a lipid bilayer. ${ }^{5}$ On the other hand, we can use the canonical ensemble average of effective acceptance ratios or reduced Boltzmann factors to reduce large fluctuations stemming from the estimation of the excess entropy. ${ }^{6}$ However, these methods are hard to apply to complex systems because of significant residual fluctuations. To solve the fluctuation problem fundamentally and obtain more stable ensemble average, we may use an acceptance ratio along with a scaling factor. ${ }^{7}$

In this work we approximately estimate the excess entropy $S^{e x}$ from the relation

$$
\frac{S^{e x}}{N k} \approx \ln \left[\left\langle s a_{R}\right\rangle_{\Delta}\right]=\ln \left[s\left\langle a_{R}\right\rangle_{\Delta}\right],
$$

where $s$ is the scaling factor and $\langle\cdots\rangle_{\Delta}$ denotes the ensemble average within a cell. Here the acceptance ratio, $a_{R}$, is written

$$
a_{R}=\left\{\begin{array}{ll}
\exp (-\Delta \phi / k T) & \text { if } \Delta \phi \geq 0 \\
1 & \text { if } \Delta \phi<0
\end{array},\right.
$$

where $\Delta \phi$ is the potential energy change which would result from the migration of a particle to a random position within the cell during computer simulation. The cell is chosen to be a cube, having a fixed volume of $V / N$, centered at the sampled particle. A particle sampled during the constant NVT molecular dynamics ${ }^{2}$ or Metropolis Monte Carlo ${ }^{2,8}$ simulation is migrated to a random position within a cell. After the potential energy of the migrated particle is calculated, the particle is replaced for the normal simulation. The excess Helmholtz free energy $A^{e x}$ is obtained by

$$
A^{e x}=U^{e x}-T S^{e x},
$$

where $U^{e x}$ is the average potential energy of the system. The scaling factor in Eq. (2) is evaluated from the relation

$$
s=\frac{\exp \left(S^{e x} / N k\right)}{\left\langle a_{R}\right\rangle_{\Delta}}=\frac{\exp \left[\left(U^{e x}-A^{e x}\right) / N k T\right]}{\left\langle a_{R}\right\rangle_{\Delta}} .
$$

The scaling factors for hard-sphere (HS) fluids and LennardJones (LJ) fluids are easily obtained using available simulation data. ${ }^{9,10}$ For the HS and LJ fluids, the scaling factors are found to be linear functions of the reduced density $\rho^{*}$ at low and intermediate densities, but the scaling factors converge at high densities. The reduced quantities $\rho^{*}$ and $T^{*}$ denote $\rho \sigma^{3}$ and $k T / \varepsilon$, respectively, where $e$ is the well depth of the LJ potential and $\sigma$ is the distance at which the potential energy passes through 0 . For the HS and LJ fluids, the scaling factors are given, respectively, by

$$
s= \begin{cases}0.55+1.5 \rho^{*} & \text { if } \rho^{*} \leq 0.8 \\ 1.75 & \text { if } \rho^{*} \geq 0.8\end{cases}
$$

and

$$
s=\left\{\begin{array}{ll}
1.4+0.75 \rho^{*} & \text { if } \rho^{*} \leq 0.8 \\
2.0 & \text { if } \rho^{*} \geq 0.8
\end{array} .\right.
$$

The method of obtaining the excess entropy from Eq. (2) will be termed the acceptance ratio (AR) method.

Monte Carlo and molecular dynamics simulations were performed for the HS and LJ fluids. All the simulations were performed with 216 molecules and about $3 \times 10^{6}$ configurations were averaged after equilibration using periodic boundary conditions. A detailed description of the esti- 
mation of $a_{R}$ can be found elsewhere. ${ }^{11}$ The restricted random search (RRS) method ${ }^{12}$ was used, along with the AR method, to avoid the quasi-ergodicity.

The excess free energies calculated using the AR method for HS fluids are compared in Figure 1 with the results of thermodynamic integration from the Carnahan-Starling equation of state. ${ }^{9}$ The excess free energies calculated using the AR method for the LJ fluids are shown in Figure 2, compared with those from thermodynamic integration ${ }^{10}$ and the Weeks-Chandler-Anderson perturbation theory. ${ }^{13} \mathrm{We}$ obtained very stable values converging to within \pm 0.01 for the excess free energies. Considering that a variety of chemical and biological processes proceed in the highdensity region, it is remarkable that the present results are very stable and in excellent agreement with the results of other calculations for both the HS systems and the LJ

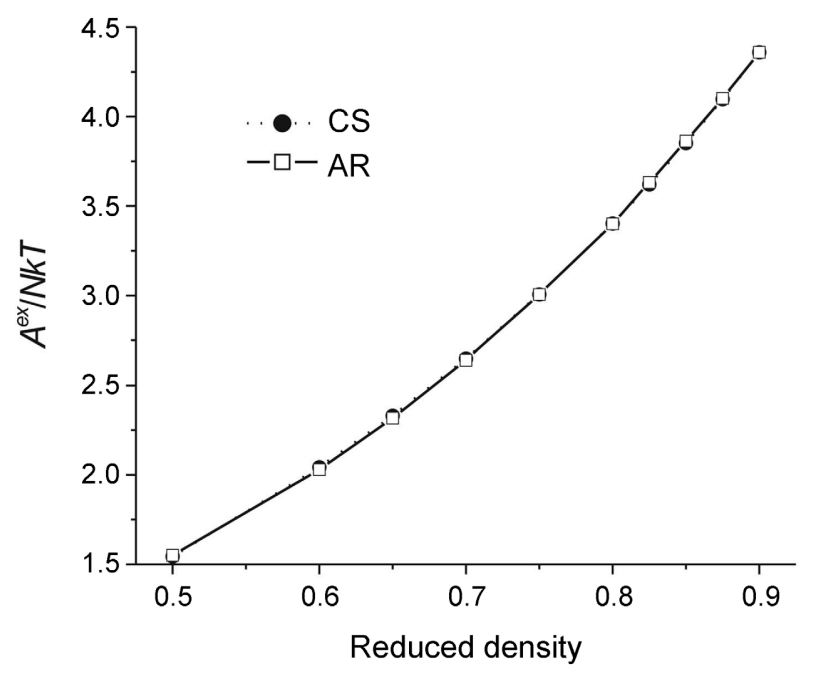

Figure 1. The excess free energies obtained by the AR method (AR) and thermodynamic integration from Carnahan-Starling equation of state (CS) for hard-sphere fluids.

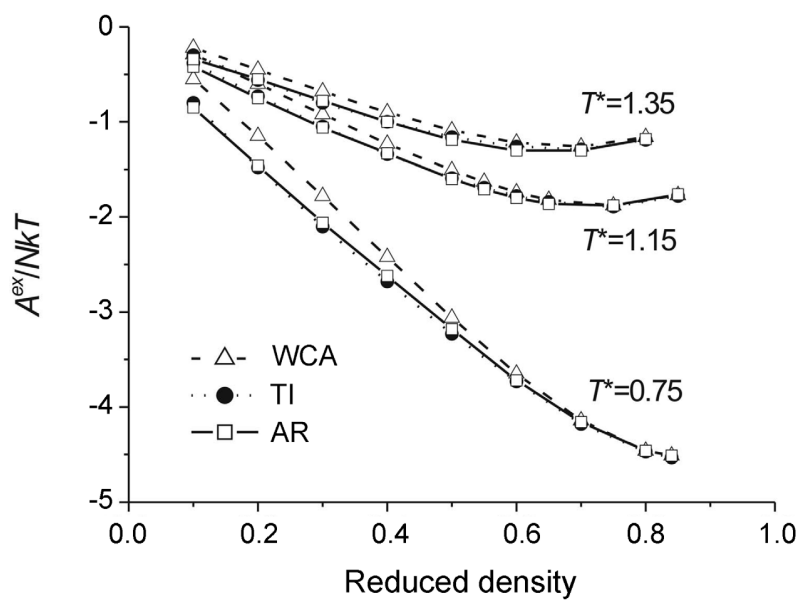

Figure 2. The excess free energies obtained using the AR method (AR), thermodynamic integration (TI), and the Weeks-ChandlerAnderson (WCA) perturbation theory for Lennard-Jones fluids on the isotherms $T^{*}=0.75,1.15$, and 1.35 . systems.

Whereas the application of the fluctuating cell model is limited to high-density phases of two-dimensional hard dumbbells, ${ }^{14}$ the AR method has been applied successfully to three-dimensional systems. Because no reference system is needed in the AR method, the excess entropy is directly calculated and the difficulties arising from the phase transitions of high-density fluids or solids can be overcome. $^{15}$ Moreover, the present method appears to be extremely efficient. The AR method requires a simulation of only $3 \times 10^{6}$ configurations to obtain the excess free energy of for a square-well fluid at $\rho^{*}=0.68$ and $T^{*}=1.29$, whereas the temperature and density scaling Monte Carlo method requires a much longer simulation with a reference state to obtain the same value. ${ }^{16}$ The quasi-ergodicity resulting from the Metropolis algorithm can be avoided using the RRS method ${ }^{12}$ or the jump-walking method. ${ }^{17}$

Considering that the converged scaling factors differ little between the HS fluids and the LJ fluids, the present method may be extensively applicable to such solutions and biological systems as their solvation free energies are largely entropic and not enthalpic, ${ }^{18}$ as the PI method was modified and applied to small molecules in a lipid bilayer. ${ }^{5}$

Acknowledgment. This work was supported by Korea Research Foundation Grant. (KRF-2003-005-C00006).

\section{References}

1. Valleau, J. P.; Torrie, G. M. In Modern Theoretical Chemistry; Berne, B. J., Ed.; Plenum: New York, 1977; Vol. 5, pp 169-194.

2. Allen, M. P.; Tildesley, D. J. Computer Simulation of Liquids; Clarendon: Oxford, 1987; pp 213-220.

3. Frenkel, D.; Smit, B. Understanding Molecular Simulation: From Algorithms to Applications, 2nd ed.; Academic: London, 2002; pp 167-200.

4. Widom, B. J. Chem. Phys. 1963, 39, 2808.

5. (a) Deitrick, G. L.; Scriven, L. E.; Davis, H. T. J. Chem. Phys. 1989, 90, 2370. (b) Jedlovszky, P.; Mezei, M. J. Am. Chem. Soc. 2000, 122,5125 .

6. (a) Hong, S. D.; Jhon, M. S. Chem. Phys. Lett. 1997, 273, 79. (b) Hong, S. D.; Yoon, B. J.; Jhon, M. S. Chem. Phys. Lett. 1992, 188 , 299. (c) Hong, S. D. Bull. Korean Chem. Soc. 2003, 24, 1351.

7. Hong, S. D.; Jang, D.-J. Chem. Lett. 2002, 31, 442.

8. Metropolis, N.; Rosenbluth, A. W.; Rosenbluth, M. N.; Teller, A. H.; Teller, E. J. Chem. Phys. 1953, 21, 1087.

9. Carnahan, N. F.; Starling, K. E. J. Chem. Phys. 1969, 51, 635.

10. (a) Levesque, D.; Verlet, L. Phys. Rev. 1969, 182, 307. (b) Hansen, J.-P.; Verlet, L. Phys. Rev. 1969, 184, 151.

11. Hong, S. D.; Jang, D.-J. Chem. Lett. 2002, 31, 946.

12. Hong, S. D.; Jhon, M. S. Chem. Phys. Lett. 1997, 267, 422.

13. Weeks, J. D.; Chandler, D.; Anderson, H. C. J. Chem. Phys. 1971, $54,5237$.

14. Gay, S. C.; Rainwater, J. C.; Beale, P. D. J. Chem. Phys. 2000, 112,9841

15. Yoon, B. J.; Hong, S. D.; Jhon, M. S.; Scheraga, H. A. Chem. Phys. Lett. 1991, 181, 73.

16. Brilliantov, N. V.; Valleau, J. P. J. Chem. Phys. 1998, 108, 1115.

17. (a) Frantz, D. D.; Freeman, D. L.; Doll, J. D. J. Chem. Phys. 1992, 97, 5713. (b) Tsai, C. J.; Jordan, K. D. J. Chem. Phys. 1993, 99, 6957.

18. Chandler, D. Nature 2002, 417, 491. 